\title{
Building a Social Newsroom Visual Analytics for Social Business Intelligence
}

\author{
Zimmerman, Chris; Wessels, Henricus T. W. J.; Vatrapu, Ravi
}

Document Version

Final published version

\section{Published in:}

Proceedings of the 2015 IEEE 19th International Enterprise Distributed Object Computing Conference Workshops and Demonstrations (EDOCW 2015)

DOI:

10.1109/EDOCW.2015.20

Publication date:

2015

\section{License}

Unspecified

Citation for published version (APA):

Zimmerman, C., Wessels, H. T. W. J., \& Vatrapu, R. (2015). Building a Social Newsroom: Visual Analytics for Social Business Intelligence. In S. Hallé, \& W. Mayer (Eds.), Proceedings of the 2015 IEEE 19th International Enterprise Distributed Object Computing Conference Workshops and Demonstrations (EDOCW 2015) (pp. 160163). [7310688] IEEE. https://doi.org/10.1109/EDOCW.2015.20

Link to publication in CBS Research Portal

\section{General rights}

Copyright and moral rights for the publications made accessible in the public portal are retained by the authors and/or other copyright owners and it is a condition of accessing publications that users recognise and abide by the legal requirements associated with these rights.

Take down policy

If you believe that this document breaches copyright please contact us (research.lib@cbs.dk) providing details, and we will remove access to the work immediately and investigate your claim. 


\section{Building a Social Newsroom: Visual Analytics for Social Business Intelligence}

\author{
Christopher J. Zimmerman ${ }^{1,2}$ \\ ${ }^{1}$ Computational Social Science Laboratory \\ Department of IT Management, \\ Copenhagen Business School \\ cz.itm@cbs.dk
}

\author{
Henricus T.W.J. Wessels ${ }^{2}$ \\ ${ }^{2}$ Mindjumpers ApS \\ Kristianiagade 8, 4th floor - 2100 \\ Copenhagen $\varnothing$, Denmark \\ erwin@mindjumpers.com
}

\author{
Ravi Vatrapu ${ }^{1,3}$ \\ ${ }^{3}$ Mobile Technology Laboratory \\ Faculty of Technology \\ Westerdals Olso School of ACT \\ vatrapu@cbs.dk
}

\begin{abstract}
Insufficient data visualization in current social media tools is hampering opportunities to make effective meaning and take decisive action from social data. This paper presents the technical architecture of a prototype tool for Social Business Intelligence (SBI) under development. Adopting an Action Design Research approach, the goal of the 'Social Newsroom' is to provide practitioners with user interfaces for leveraging such affordances. The construction of specific interfaces is detailed including monitoring dashboards and insights pillars for visual analytics.
\end{abstract}

Keywords- Social Business, Data Science, Visual Analytics, Business Intelligence, Social Media Analytics, Affordances

\section{INTRODUCTION}

Today, businesses are utilizing social media as part of their strategy for communicating with and understanding the behaviors of their consumers. The research project, in its entirety, seeks to derive business value from social data by designing and developing a series of dashboards for those who struggle to interpret and keep up with the social data created around a brand and marketing campaign.

As we discuss in the next few sections, the Social Newsroom tool is theoretically informed (in the theory and conceptual model of social data) [1], historically aware (with reference to commercial tools and research prototypes) [2], technically robust and sound (in terms of visual analytics) [3], methodologically rigorous (following Action Design Research) [4], and empirically validated (in real-world social media marketing campaigns and trainee evaluations) [5]. The tool aims to realize novel exploratory social data visualizations for rapid discovery of emergent themes while also providing support for established metrics in marketing with efficient blends of online conversations, communities, and campaign objectives.

The remainder of the paper is organized as follows. We first outline the foundation of prototype development with focus on the main theoretical perspectives guiding the research followed by the corresponding methodological approach. Next we provide a system overview that explains the technical architecture needed to pursue such inquiries. A summary of the entire tool design is subsequently put forward highlighting each component. The Social Newsroom tool is accessed by practitioners at baboon.mindjumpers.com and a full video demo is at vimeo.com/datavist/socialnewsroom (pwd: sbidemo).

Our research project is building on and extending on the currently available social media analytics methods and tools, to identify new approaches that contribute to both the academic and business aspects of 'Social Business Intelligence'[6]. The developmental objective of this project involves going beyond the standard metrics and visualizations used in social media reporting to explore in greater depth the nature of a company's online advocates and gain a holistic understanding of what the company's brand looks like in the eyes of its consumers.

Regarding theoretical foundations, our tool is informed by the theory and conceptual model of social data which distinguishes between social graph and social text and suggest that social media analytics can be undertaken in two main ways: "Social Graph Analytics" and "Social Text Analytics" [7]. Social graph analytics is concerned with the structure of the relationships emerging from social media use. Social text analytics is more concerned with the substantive nature of the interactions.

Regarding historical awareness, a systematic review of existing research prototypes and commercial tools can be found in [2]. Our tool addresses a research gap in the extant academic literature (lack of design principles for social business intelligence dashboards) and targets a features gap in commercial tools and research prototypes (lack of dashboards that integrate real-time monitoring and post hoc reporting of social media marketing campaigns). Next, we discuss the technical and methodological dimensions of the Social Newsroom.

\section{DESIGN OF THE IT-ARTEFACT}

\section{A. Problem Statement \& Intended Target Groups}

One major practical challenge for social media marketers has been the need for data to support decision-making in the creation and curation of real-time content and community management. Creating a discourse through planned communication and strategic use of language is a way of attempting to lead followers and affect organizational culture [8]. Motivating consumer engagement through content creation must be undertaken with more uncertainty since language is socially constructed and context specific, and hence will change more online from one person to the other, than it would in physical and organizational network contexts. The design of a social media dashboard to that end has led to several outcomes in the form of dedicated interface designs which are subsequently described in detail in the following prototype demo. Considering these use cases, the target end users include social media managers, analysts, 
marketers, chief marketing officers, and members of social media teams throughout business organization structures.

Existing state-of-the-art social media tools already provide data collection, aggregation and analysis into KPIs. However when represented in dashboards, they lack visualizations that can facilitate meaning making opportunities and action taking possibilities for business intelligence. The visualization of social media data and subsequent monthly reporting therefore suffer from a lack of standards and design principles. Current tools either require data science support with complementary competencies or supplementary tools to design, develop, evaluate and use the actionable data from social channels.

\section{B. Action Design Research (ADR) Approach}

Current development of the platform is taking place within both a research environment and a social media marketing agency. The research methodology has employed Action Design Research (ADR) as a practical way of eliciting needs from the industry in order to identify what new technological affordances are possible and what level of value is brought via visualization. According to Sein et al, "traditional design science does not fully recognize the role of organizational context in shaping the design as well as shaping the deployed artifact... They value technological rigor at the cost of organizational relevance, and fail to recognize that the artifact emerges from interaction with the organizational context even when its initial design is guided by the researchers' intent."[4] Sein et al. also distinguish that the Information Systems field has a broad consensus that seeks to respond to a dual mission of making theoretical contributions while solving (expected) practitioner problems, citing Benbasat and Zmud 1999; Iivari 2003; Rosemann and Vessey 2008 [4]. For such reasons an ADR approach has been the ongoing methodology for the design, development and evaluation of the tool. The initial design phase was largely based on learning tools absent from the social media tool landscape, as well as concrete reporting needs for a celebrity campaign led by Skype with fashion designer Victoria Beckham. The development phase of the tool was subsequently shaped by successive product launch campaigns by the electronics manufacturer Bang \& Olufsen in 2014 and 2015. The product launch events have directly led to realtime monitoring dashboards with elements elicited directly from the social media response teams who would subsequently use them for interaction with the public. Further case details of the tool's control room launch setup can be found in a newspaper article (in Danish) [5]. A subsequent evaluation phase now includes additional brand channel admin accounts (Facebook, Twitter, YouTube, Instagram) for five other clients that are being tracked by the tool. Brand managers who serve these clients can now offer early and informal evaluation on the tool's effectiveness, while further surveys and eye tracking experiments will pinpoint feedback for precise feature usage and visual comprehension. Since design and development of this tool started concurrently, the initial and ongoing cycle of ADR has been crucial in order to detect the needs of target users. Most importantly the "build and then evaluate" cycles have led to constant elicitation of user needs for the artifact in development.

\section{Technical Architecture}

Figure 1 presents a technical schematic of the current Social Newsroom prototype. Technically, there are four main components to the data architecture: the database, a set of data gathering \& processing scripts, an admin site and a front-end site.

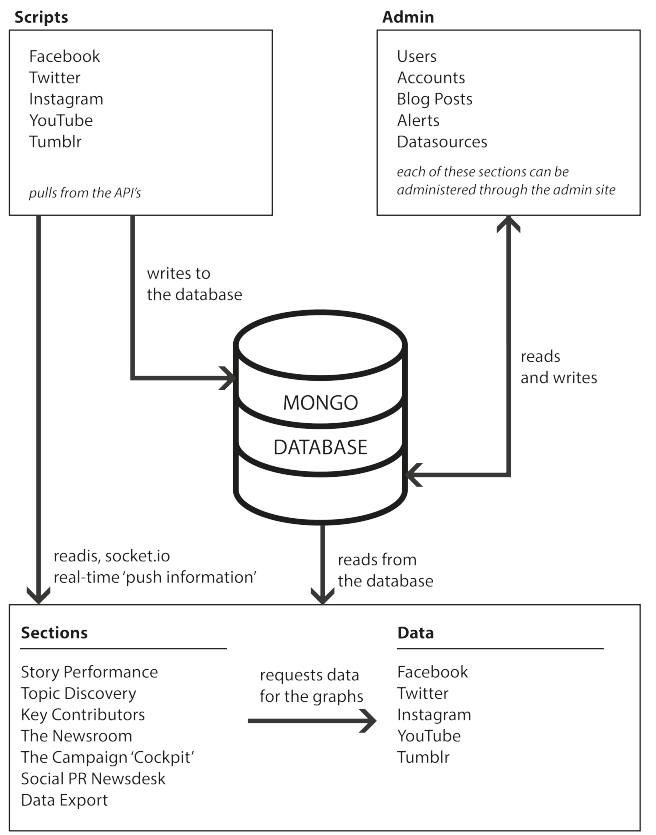

Figure 1: Schematic Diagram of Social Data for the design project. A NoSQL database stores data gathered from social platform APIs. A NodeJS back-end fetches data from each platform and optimizes it before storing social data. JavaScript modules then fetch periodically without missing any new data from the social platform's API.

Database - The database is MongoDB, a NoSQL database that provides the flexibility to adapt the structure and performance to be able to run the entire system [9]. MongoDB has the capacity to grow to a cluster of machines but currently this is a single database server. Structurally, there are two separate databases: one for data, with collections per data item (Facebook posts, tweets, etc.), and one for the site configurations (users, etc.).

Scripts, Redis \& Socket.io - Data gathering scripts are written in Node.js, which allows for rapid iterations and development of a growing code base to connect to each individual social media platform [10]. The scripts themselves are broken down into single functionality scripts with modules per social media platform providing the interaction with the Facebook, Twitter, Instagram, YouTube and Tumblr APIs, as well as for accessing RSS feeds. For example, a single script currently fetches new posts by Facebook pages being monitored. The majority are pull-APIs in which information is requested. However Twitter's streaming APIs allows subscriptions to a set of searches that provide push information when any new 
tweet matches a given search string. These queries are thus pushed to the data infrastructure in real time. Whenever the scripts discover new interaction items (be that a Facebook post or comment, or a tweet, etc. etc.) a message is published through Redis, a rather unique system which offers functionalities for storing key-value pairs [11]. This project leverages Redis for publishing messages throughout our social data system using PubSub [12]. The 'Campaign Overview' dashboard, for example, receives updates from the server from an alerter script. This script receives new items from Redis and passes them through Socket.io to any and every browser that is currently displaying the newsroom section [13]. A final modular script supports notifications, e-mails to be sent out when a certain new item (Facebook post, tweet, youtube video, etc) matches a certain condition.

Admin Site - The admin site is a Model-view-controller (MVC)-based PHP site, which allows admins to manage access to the front-end site, set up topic profiles for these users (connections to Facebook pages, keywords, etc) and configure various other parts of the system.

Front-end Site - The front-end is written in PHP and is a single-page application, with the individual sections of the site (designs later referred to as 'insights pillars' and 'monitoring dashboards') loaded in separately. Data is loaded through AJAX, originating from an API (built in PHP) that fetches data from Mongo and passes them to the front-end (using JSON). The newsroom pillar is the only pillar which currently receives real-time updates through, as mentioned, socket.io. Finally, all dashboard charts are drawn in Scalable Vector Graphics (SVG) using the venerable D3 library [14], allowing custom graphics for interactive dashboard experiences, such as the dashboard map displaying real-time conversation volumes [15].

\section{DESIGN OF THE PROTOTYPE}

The artifact design has thus far combined six individual interfaces that serve specific social business purposes for practitioners. They are each designed with a primary visualization and supported by supplemental gauges to provide additional contextual background. The six interfaces can be separated into three "pillars" for insights and three "dashboards" for monitoring. The following demonstration of interfaces for the Social Newsroom BI tool (Table 1) subsequently details each individual design and purpose. These interactive displays can be sharply contrasted with commonplace static examples, such as word clouds for topic discovery, line graphs for performance, and tables ranking people. A global toolbar allows for users to both switch between brands as well as isolate any time frame with a date-picker.

\section{Insights Pillars (P1 - P3)}

The three pillars for insights are intended to give the practitioner an ability to learn about story performance, important people and relevant topics respectively with interactive charts. This allows them to follow an information scent, or insight, and then have the possibility of leveraging their intuitions to inform other investigations within the other interfaces. The user thus has a hypothetical opportunity to shift content strategy in posting and re-assess performance gauges in a cyclical usage pattern of the dashboard. An information foraging path is evaluated during and after deployments.

\section{Performance Insights Pillar I (Story Performance)}

In order to continuously improve and create more engaging and relevant content for a given community, the case company needs to measure how the content is performing and to analyze the reasons why content is (or is not) performing. The Story Performance pillar is a selfreflective view of the campaign's own posting performance on Facebook, Twitter and Instagram, blended together using multi-dimensional visual representations. Currently this displays how well individual stories, themes, and timings are being received by social media communities along four dimensions, including ActionReaction Ratios, Affinity, Amplification, and Commentary magnitude. A primary bubble graph portrays amplification and affinity metrics of stories on either access while bubble size reflects the amplitude of story commentary. The ultimate success of each piece of content is immediately visible, while clustering effects can shed light on patterns of content type, topic, and language. Finally, a glanceable calendar distribution contrasts proportions between publishing actions and fan reactions.

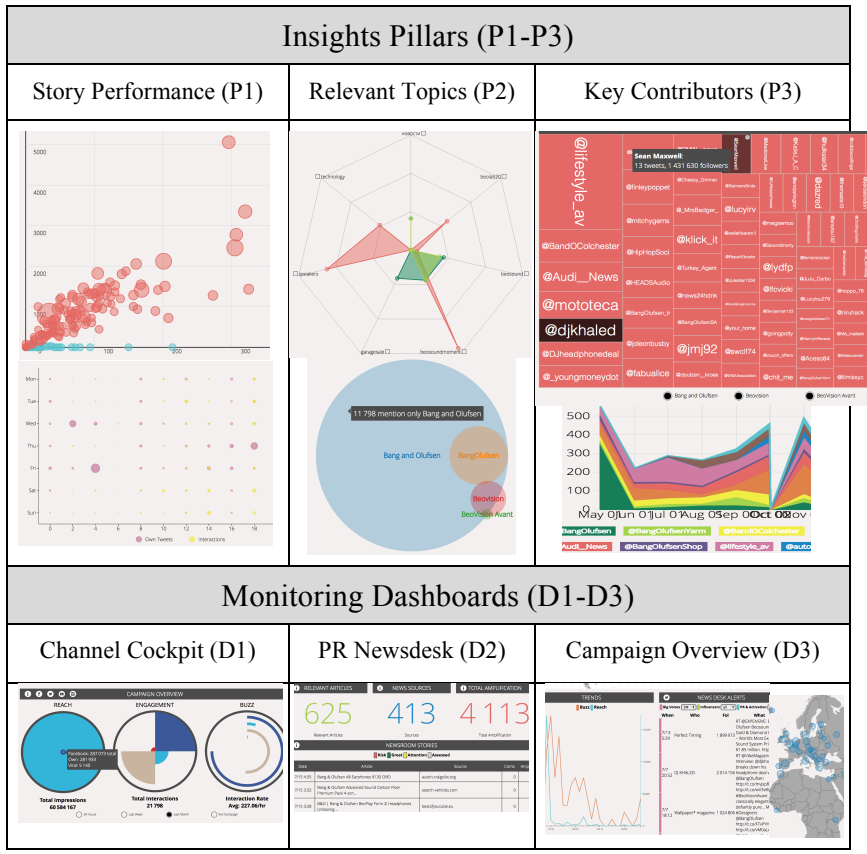

Table 1. The User Interface (UI) presents data from the database via six interfaces, each holding groups of complimentary charts built with the D3 JavaScript library.

\section{Social Text Insights Pillar II (Topic Discovery)}

In order to be able to produce relevant, real-time content for the brands it supports, a company monitors trending topics and uncovers new topics from within its community. This information may also potentially inform campaigns, services or even the physical design of future products. Such socially-constructed folksonomies of a brand offer an unbiased definition by the community. This dashboard was designed to intuitively act as a radar for 
common words that exist around several brands or topics of interest. The interactive radar graph leverages language text from user-generated posts to identify related topics that revolve around given keywords or brands. Topics of intersest such as a brand, competitor or celebrity sponsor are mined to allow the tool to identify related words with the greatest frequency. When several topics are selected simultaneously, the keywords on the radar are overlapping volumes of harmonized interest areas. Each color-coded language footprint can also be manipulated to filter out irrelevant terms that surface, drilling-down to the most relevant representation for analysis. A stream of posts containing both keyword and topics is also accessible to provide context to the user. The design also includes contextual graphs. Venn diagrams of post repositories reveal total volumes/overlaps embedded in a conversation.

Social Graph Insights Pillar III (Detect Key Contributors) In order to create relevant content it is also necessary to gain an in-depth understanding of the community. By isolating clusters, interventions can alter the course of a conversation as sentiments are disseminated through the network graph of the brand page. The Key Contributors dashboard includes a selection of tools that show individual users who are talking about a chosen brand, campaign, or topic as well as how much they are talking (or any combination thereof). Size and shading allow quick identification of the most important people based on their degree of involvement and following. Additional graphs show share of voice and volumes of hashtag usage.

\section{Monitoring Dashboards (D1 - D3)}

A second set of dashboards serves the real-time needs of a campaign newsroom that must constantly monitor exposure on social channels.

The 'Cockpit' - Dashboard I: The campaign cockpit design currently enables the manager or business leader to take the temperature of the current brand situation on four channels in the same dashboard (Facebook, Twitter, YouTube and Instagram) to monitor performance on a high level. Social media channels are color-coded within the gauges to contrast standard performance metrics within three combinable, cross-channel dimensions: Reach, Engagement and Buzz.

The Social PR Newsdesk - Dashboard II: Social media tools ignore web articles outside of social media and their consequent share counts on social channels. A newsdesk repository was thus built using pre-directed RSS feeds from Google Alerts. This piping offers automatic population of relevant news stories to practitioners who manually use the newsdesk to mark-up each article with context (sentiment, number shares per channel) and flag content for taking action by community managers.

The Campaign Overview - Dashboard III: In further listening to these end-users, a final Social Newsroom interface was established that combines a three-part overview of campaign exposure. A primary map display reflects global volume of campaign mentions that are currently emanating around the world. This can be toggled from user-selected historical windows to 'live mode'. A time series graph plots total volume of posts (mentions) and a dual-axis for corresponding reach levels. Finally a timeline of alerts for posts from influential people (with high follower levels) or key influencers such as journalists and bloggers from press materials and PR outreach. The combined effect provides a contextual interface where a user in a control room setting can notice a spike in volume, refer to the map for its origination, and also see that any major actors (celebrities, etc.) in the alert feed who mentioned campaign or brand topics. The alert feed also includes an audio cue to grabs the user's attention (who is often multitasking across screens and reports) to take immediate notice and take direct action.

\section{CONCLUDING REMARKS}

This tool prototype summary illustrates that the design of the Social Newsroom is not simply product development, but an IT artifact that seeks to be theoretically informed, methodologically built and empirically tested towards facilitating social business intelligence opportunities. Technically, the entire system is set up to be broken up into discreet components than can run on separate (clusters of) servers. In order to be able to process larger volumes of data, the data gathering scripts are designed to be run on separate servers, and allow for concurrent operation (so that multiple processes in parallel could gather the same type of data). In terms of storage, MongoDB is designed to be able to scale to substantial clusters without any problems. In order to be able to further enrich data, the storage system is flexible in storage and allows for additional keys to be added to records without any problems. In practice, the resulting interfaces offer unique affordances that harmonize datapoints across social media channels into displays that show custom blends of the conversation and community. Trial licenses to real-world companies have been granted during development and will lead to further empirical testing within real-world contexts; that is to say by the actions of real-world users generating genuine usage data, soon to be verified in lab simulations and validated by further practitioner cases.

\section{References}

[1] R. Vatrapu, R. Mukkamala, and A. Hussain, "Social set analysis: four demonstrative case studies," Proc. 2015 ..., 2015.

[2] C. Zimmerman, "A Classification of the Socio-Technical Affordances of Commercial and Research Prototype Tools for Social Business Intelligence (In Print)," in The 38th Information Systems Research Conference in Scandinavia (IRIS38), 2015, p. 18.

[3] C. Ware, Information visualization: perception for design. Waltham, MA, 2012

[4] M. Sein, O. Henfridsson, S. Purao, M. Rossi, and R. Lindgren, "Action design research," 2011.

[5] S. Ingemann, "B\&O-kampagne flot fra start," Markedsføring, 12-May2014 .

[6] B. Dinter and A. Lorenz, "Social Business Intelligence: a Literature Review and Research Agenda," 2012.

[7] R. Vatrapu, "Understanding social business," Emerg. Dimens. Technol. Manag., 2013.

[8] M. Alvesson, "Organizational Culture," Handb. Organ. Cult. Clim., 2011.

"MongoDB." [Online]. Available: https://www.mongodb.org/.

"Node.js." [Online]. Available: https://nodejs.org/.

"Redis." [Online]. Available: http://redis.io/.

"Pub/Sub - Redis." [Online]. Available: http://redis.io/topics/pubsub "Socket.IO." [Online]. Available: http://socket.io/.

"D3.js - Data-Driven Documents." [Online]. Available: http://d3js.org/. M. Bostock, V. Ogievetsky, and J. Heer, " $\mathrm{D}^{3}$ data-driven documents," Vis. Comput. ..., 2011. 